\title{
PHYTOPLANKTON BASED ASSESSMENT OF ECOLOGICAL STATUS OF BULGARIAN LAKES AND COMPARISON OF METRICS WITHIN THE WATER FRAMEWORK DIRECTIVE
}

\author{
Belkinova, D. ${ }^{1}$ - PAdisÁK, J. ${ }^{2}$ - GeCheva, G. ${ }^{1}$ - CheshmedJIEV, S. ${ }^{1}$ \\ ${ }^{1}$ University of Plovdiv, Faculty of Biology \\ Tsar Asen Str. 24, 4000 Plovdiv, Bulgaria \\ (phone: +359-32-261-507; fax: +359-32-261-566) \\ ${ }^{2}$ University of Pannonia, Department of Limnology \\ Egyetem ut 10, 8200 Veszprém, Hungary \\ (phone: +36-88-624-747; fax: +36-88-624-008) \\ * Corresponding author \\ e-mail:detbel@mail.bg \\ (Received $8^{\text {th }}$ Nov 2012 ; accepted $22^{\text {nd }}$ July 2014)
}

\begin{abstract}
Taxonomic composition and abundance of summer phytoplankton were studied in 78 lakes in Bulgaria. The Hungarian Assemblage index was applied for the first time on various Bulgarian lake types and 27 phytoplankton functional groups were identified. The dominant functional groups differed based on lake type and trophic status. Functional groups' frequency distribution showed that high relative abundance of assemblages $\mathrm{L}_{0}, \mathrm{Y}, \mathrm{MP}, \mathrm{N}, \mathrm{E}, \mathrm{X} 3, \mathrm{X}_{\mathrm{ph}}$ indicated high or good status, regardless of lake type. Simultaneously undesirable assemblages, linked to the declined water quality, were $\mathrm{L}_{\mathrm{M}}, \mathrm{J}, \mathrm{M}, \mathrm{S} 1$ and $\mathrm{H} 1$. We also examined the relationship between the Assemblage index and a number of phytoplankton metrics: Total biomass, \% Cyanobacteria, Transparensy, Chlorophyll $a$, Algal bloom and Algae Group Index. The two applied indices (Hungarian Assemblage index and Algae Group index) were highly correlated $(\mathrm{p}<0.01)$. The Assemblage index had also strong relationship $(\mathrm{p}<0.001)$ with Total biomass, $\%$ Cyanobacteria, Chlorophyll $a$, as well as with the assessed overall ecological status. Assemblage index and Algae Group Index resulted in similar assessment and were applicable to all three Alpine and mountain, Deep semimountain and Small and middle sized lowland lake groups. Assemblage index is recommended especially for lentic ecosystems in extreme cases, e. g. hyperhaline lakes, for its flexibility and more adequate assessment.
\end{abstract}

Keywords: Assemblage index, Q index, Algae Group Index, Functional groups, Biomass

\section{Introduction}

Studies on phytoplankton communities and their relationship with water quality status have a long history (Padisák et al., 2006; Cheshmedjiev et al., 2010a). A number of phytoplankton based indices have been developed for the purpose of Water Framework Directive (WFD). Among them six were applied in South Europe: Algae Group Index (AGI, Catálan Index) in Spain and Italy (Agència Catalana de l'Aigua, 2003; Marchetto et al., 2009); ITP or Barbe Index in France (Philippe et al., 2003); Brettum index in Austria (Dokulil and Teubner, 2006); Phytoplankton Trophic Index (PTI) in Italy (Salmaso et al., 2006); Mediterranean Phytoplankton Trophic Index (MedPTI) in Italy (Marchetto et al., 2009), and Assemblage index (Q index) in Spain (Becker et al., 2010). According to Marchetto et al. (2009) indices can be differentiated into two groups. The first one (Brettum and MedPTI), based on species trophic preference, evaluates abundance of each taxa and adjudges trophic points/values. The second group (AGI and Q) applies percent biomass share of particular algae. 
Assemblage index was developed for the assessment of lakes in Hungary within the WFD (Padisák et al., 2006). It requires species level determination and combines relative share of species assemblages with factor numbers for particular lake types (Becker et al., 2010). Phytoplankton functional group (FG) concept outlined in Reynolds et al. (2002) was followed for "functional groups" establishment, as well as physiological, morphological and ecological attributes of the species that may potentially dominate or co-dominate in a particular type water body. Subsequently, the approach was further developed (Padisák et al., 2003; 2009). At present, 38 FGs are described, identified by numeric character codes (codons). The Q index is a reliable instrument to assess ecological status and trends (Padisák et al., 2006; Becker et al., 2010). Besides Hungarian lakes, Q index was also successfully tested at shallow tropical, deep subtropical and deep Mediterranean reservoirs (Crossetti and Bicudo, 2008; Becker et al., 2009; 2010). A major advantage of Q index is that unlike other indices, it reflects general anthropogenic pressure.

Algae Group Index (AGI) is the first index developed particularly for Mediterranean sites (Agència Catalana de l'Aigua, 2003) and was calibrated on the basis of Spanish reservoirs data. Algae Group Index was founded on proportion between biomass of two algae units. In view of high levels of taxa determination, AGI should be applied carefully, because its algal groups include species with different ecological preferences (Marchetto et al., 2009).

New phytoplankton functional classifications, based on a morphological approach, were reported recently (Salmaso and Padisák, 2007; Kruk et al., 2010). Classification of Salmaso and Padisák (2007) is based on morphological and functional criteria (size and shape, mobility, potential mixotrophy, buoyancy) and divides phytoplankton into 31 morpho-functional groups (MFGs) with different adaptive strategies. Classification of Kruk et al. (2010) is clearly only morphological and seven morphologically based functional groups (MBFGs) are differentiated. Selected morphological characteristics correlated well with functional properties, such as growth and sinking rate, vulnerability to consumption and also with the population size and biomass attained in the field. Among the above concepts, the FG concept (within Q index) and MFG concept within Functional Traits Index (FTI) (Phillips et al., 2011) are elaborated in the context of WFD.

Our study had three main aims. Firstly, we attempted to apply the Q index for assessment of the water bodies from existed lake types in Bulgaria according to the accepted typology in 2009. Moreover, the Q index was tested on water bodies significantly differentiated by origin, altitude, salinity and mixing and stratification. Secondly, we examined the relationships between several existing phytoplankton metrics to explore their potential for assessment of water bodies from existed lake types in Bulgaria, among them specific ecosystems. Thirdly, we evaluated the overall ecological status of selected lakes.

The research was a logical continuation of previously published results on revision of typology and ecological status/potential of the lakes in Bulgaria (Cheshmedjiev et al., 2010a; 2010b). 


\section{Materials and methods}

\section{Study area and sites description}

Seventy-eight lakes were studied during 2009 in Bulgaria (Fig. 1). The water bodies differed in altitude characteristics (from 0 to $2375 \mathrm{~m}$ a.s.1.), origin (natural, artificial, glacial, tectonic, karst, liman, lagoon), morphometry (all classes), mixing and stratification (glacial monomictic, shallow polymictic and deep dimictic) and trophic state (from ultra oligotrophic to hypertrophic).

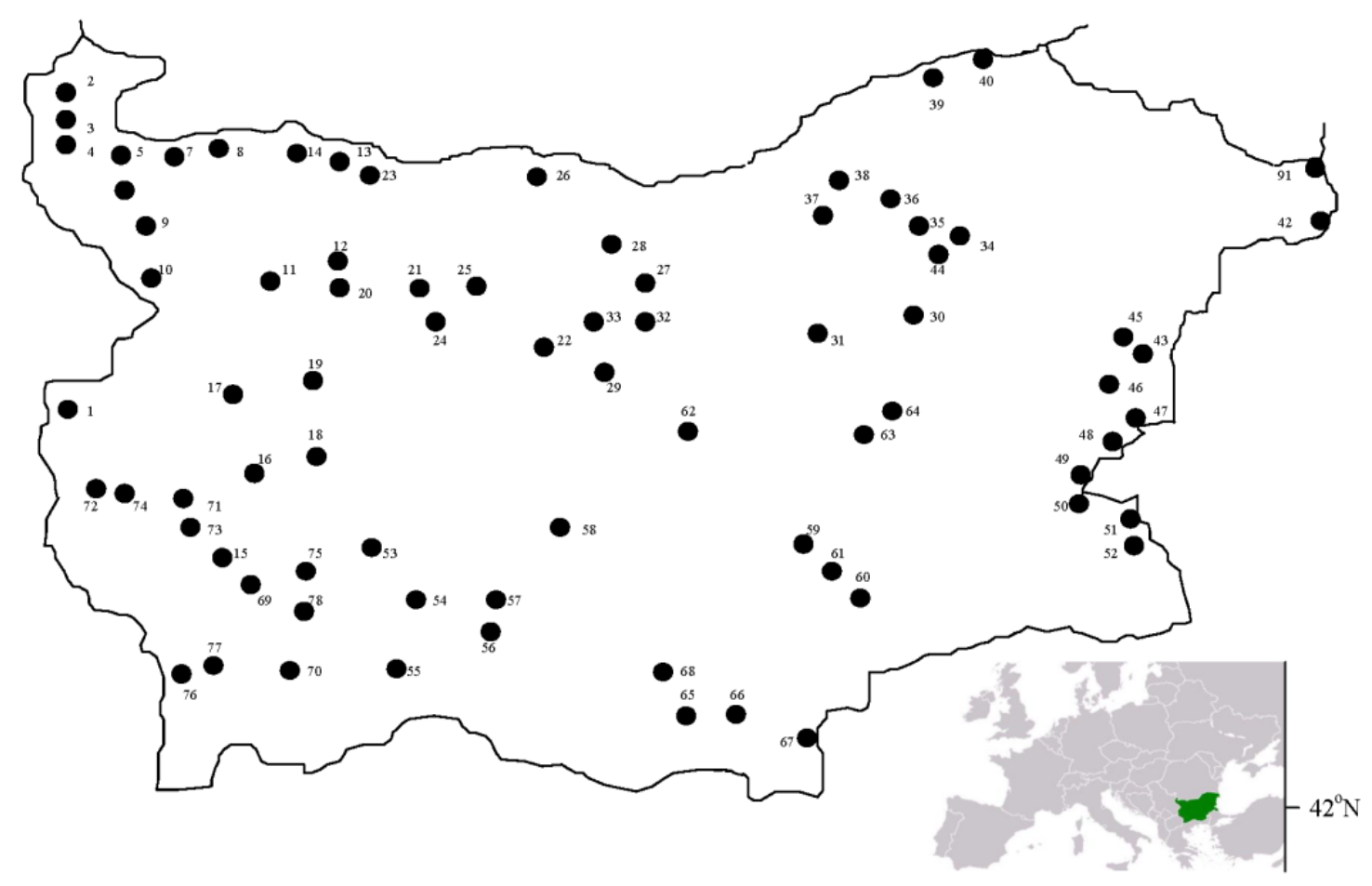

Figure 1. Location of the studied lakes. Legend (national lake type written in parenthesis): 1 Yarlovtsi Reservoir (L2); 2 - Kula Reservoir (L12); 3 - Poletkovtsi Reservoir (L12); 4 - Rabisha Reservoir (L4); 5 - Drenovets Reservoir (L16); 6 - Hr. Smirnenski Reservoir (Lomtsi) (L16); 7 -

Rasovo Reservoir (L16); 8 - Kovachitsa Reservoir (L16); 9 - Ogosta Reservoir (L14); 10 Srechenska bara Reservoir (L2); 11 - Dabnika Reservoir (L16); 12 - Tri kladentsi Reservoir (L16); 13 - Barsina Reservoir (L16); 14 - Asparuchov val Reservoir (L16); 15 - Beli Iskar Reservoir (L1); 16 - Iskar Reservoir (L11); 17 - Pancharevo Reservoir (L12); 18 - Ognyanovo Reservoir (L2); 19 - Bebresh Reservoir (L2); 20 - Devets Reservoir (L16); 21 - Enitsa Reservoir (L16); 22 - Sopot Reservoir (L12); 23 - Kruchovitsa Reservoir (L16); 24 - Telish Reservoir L16); 25 - Gorni Dabnik Reservoir (L14); 26 - Valchovets Reservoir (L16); 27 - Alexandrovo Reservoir (L16); 28 - Kamenets Reservoir (L16); 29 - Hr. Smirnenski Reservoir (Gabrovo) (L2); 30 - Yastrebino Reservoir (L12); 31 - Yovkovtsi Reservoir (L2); 32 - Al. Stambolijski Reservoir (L11); 33 - Krapets Reservoir (L12); 34 - Beli Lom Reservoir (L12); 35 - Lomtsi Reservoir (L12); 36 - Kavatsite Reservoir (L12); 37 - Boika Reservoir (L12); 38 - Baniska Reservoir (L12); 39 - Antimovo Reservoir (L16); 40 - Srebarna Lake (L5); 41 - Durankulak swamp (L7); 42 - Shabla lake (L7); 43 - Eleshnitsa Reservoir (L12); 44 - Saedinenie Reservoir (L12); 45 - Tsonevo Reservoir (L14); 46 - Acheloy Reservoir (L16); 47 - Poroy Reservoir (L16); 48 - Pomorijsko Lake (L10); 49 - Atanasovsko Lake (L10); 50 - Mandra-east Reservoir (L7); 51

- Alepu Lake (L8); 52 - Yasna polyana Reservoir (L12); 53 - Belmeken Reservoir (L13); 54 Batak Reservoir (L3); 55 - Toshkov chark Reservoir (L3); 56 - Vacha Reservoir (L11); 57 Krichim Reservoir (L11); 58 - Pyasachnik Reservoir (L15); 59 - Daskal Atanasovo Reservoir 
(L12); 60 - Ovchi kladenets Reservoir (L12); 61 - Ovcharitsa Reservoir (L12); 62 - Koprinka Reservoir (L11); 63 - Zhrebchevo Reservoir (L11); 64 - Asenovets Reservoir (L13); 65 Kardzhali Reservoir (L11); 66 - Studen kladenets Reservoir (L11); 67 - Ivaylovgrad Reservoir (L11); 68 - Borovitsa Reservoir (L13); 69 - Redzhepsko Lake (L1); 70 - Bezbog Lake (L1); 71 Studena Reservoir (L3); 72 - Pchelina Reservoir (L4); 73 - Dyakovo Reservoir (L13); 74 Choklyovo marshland (L4); 75 - Chernoto Lake (L1); 76 - Stoykovtsi Reservoir (L13); 77 Bistraka Lake (L6); 78 - Gyorgiysko Lake (L1)

\section{National typology}

Bulgaria belongs to ecoregions № 12 Pontic province and № 7 Eastern Balkans according Appendix № XI, map A of Water Framework Directive 2000/60/EC (EC Parliament and Coincil, 2000). Lake typology in Bulgaria was based on the obligatory factors (4 altitude zones, size typology based on surface area, depth and geology) and optional factors (mixing characteristics, salinity, residence time, presence of profundal zone). Seventeen lake types were identified, among them seven reservoir types, represented by heavily modified and artificial water bodies without any natural lake template within the country or region (Cheshmedjiev et al., 2010b). There are a small number of natural lakes in the country, most of them under srong anthropogenic impact. Four coastal lake types with various salinity (from freshwater $<0.5 \%$ to hyperhaline $>40 \%$ ) have been reviewed as transitional waters.

\section{Sample collection}

Integrated sampling from the deepest lake zone was carried out by Ruttner batometer during July-September 2009. The depth of the euphotic zone, was defined as 2.7 times the Secchi depth (Cole, 1994). Fixed median horisonts were sampled in alpine lakes due to their high transparency $(0 \mathrm{~m}, 1 \mathrm{~m}, 2.5 \mathrm{~m}, 5 \mathrm{~m}, 7.5 \mathrm{~m}, 10 \mathrm{~m}$ and $15 \mathrm{~m})$, whereas the whole water column was collected in shallow polymictic lakes $(<3.0 \mathrm{~m})$. Physicochemical parameters of lake water: temperature, transparency according to Secchi, electrical conductivity and $\mathrm{pH}$ were measured in situ. Additionally $\mathrm{NH}_{4}-\mathrm{N}, \mathrm{NO}_{2}-\mathrm{N}$, $\mathrm{NO}_{3}-\mathrm{N}$, soluble reactive phosphorus (SRP: $\mathrm{P}-\mathrm{PO}_{4}$ ), total nitrogen and phosphorus were analyzed just after sampling according to Strickland and Parsons (1972). Chlorophyll $a$ concentration was determined spectrophotometrically, after filtration through GF/B Whatman glass fiber filter and extraction in $90 \%$ acetone, following Jeffrey and Humphrey (1975).

\section{Phytoplankton analysis}

Phytoplankton taxonomic composition was determined by a light microscope Amplival $(1000 \mathrm{x})$ on live and formalin-fixed samples. Phytoplankton was counted at $400 x$ using an inverted microscope following the method of Utermöhl (1958). For numerous species, at least 100 specimens were counted (Lund et al., 1958). The units (cells, colonies or filaments) were counted in random plots. Biovolume was determined following the geometric forms formulas (Hillebrand et al., 1999). Transfomation of biovolume into biomass was done by $1 \mathrm{~mm}^{3} \mathrm{l}^{-1}=1 \mathrm{mg} \mathrm{l}^{-1}$ (Wetzel and Likens, 2000). Total biovolume (biomass) at each sample was presented as a sum of biovolume of all taxa. The ecological status assessment was based on 5 main (total phytoplankton biomass ( $\left.\mathrm{mg} \mathrm{l}^{-1}\right)$; Assemblage index; Algae Group Index, transparency according to Secchi (m), chlorophyll $a\left(\mu \mathrm{g} \mathrm{l}^{-1}\right)$ ) and 3 additional metrics (\% Cyanobacteria (in biomass); "bloom" (intensity) and presence of "bloom" toxic species (Microcystis, 
Aphanizomenon, Cylindrospermopsis and others). The intensity of the phytoplankton „bloom” was assessed on the basis of total biomass on a 5-degree scale, modified after Sirenko and Gavrilenko (1978): I degree $\leq 2.5 \mathrm{mg} \mathrm{l}^{-1}$; II degree: $2.5 \div 10 \mathrm{mg} \mathrm{l}^{-1}$; III degree: $10 \div 500 \mathrm{mg} \mathrm{l}^{-1}$; IV degree: $500 \div 5000 \mathrm{mg} \mathrm{l}^{-1}$; V degree (,hyperbloom”) $>5000$ $\mathrm{mg} \mathrm{l}^{-1}$. In calculating \% Cyanobacteria, some species/genus for oligotrophic waters have been excluded, focusing on toxic species and eutrophic indicators (according WFD Intercalibration technical report, Part 2 - Lakes, Section 3 - Phytoplankton biomass metrics Annexes).

\section{Phytoplankton-based Indices}

Assemblage index was calculated after Padisák et al. (2006) for a selection of 19 lakes, representing $25 \%$ of all studied lakes and allocated to 4 representative groups. Water bodies from dominant type within the groups formed of lakes in identical ecological status were selected, whereas representative water bodies into different classes of ecological quality were chosen in the rest of the groups. Q index accounted relative share of FGs into the total biomass and factor number $(F)$ determined for each FG in each lake group.

The Algae Group Index (Catálan Index) based on the percentage of biovolume of the algae groups was calculated following the formula (Agència Catalana de l'Aigua, 2003).

\section{Classification system for ecological status according to phytoplankton}

Two different scales for the basic metrics (Table 1 and Table 2) have been used in the ecological assessment and interpretation of phytoplankton data, modified for the relevant lake types in Bulgaria. The scale for AGI was modified after WFD Intercalibration technical report, Part 2 - Lakes, Section 3 - Phytoplankton composition metrics (2008); those for Chlorophyll $a$ and transparency were after Cardoso (2001). Q index scale followed Padisáк et al. (2006).

Table 1. Oligotrophic type of , lakes" (L1, L2, L3, L11, L12, L13) - classification system.

\begin{tabular}{|c|c|c|c|c|c|c|c|c|}
\hline $\begin{array}{l}\text { EQR } \\
\text { (AGI) }\end{array}$ & $\begin{array}{c}\text { Total } \\
\text { biomass } \\
\left(\mathrm{mg} \mathrm{l}^{-1}\right)\end{array}$ & $\mathbf{Q}$ & AGI & $\begin{array}{l}\text { Transp } \\
\text { arency } \\
(\mathbf{m})\end{array}$ & $\begin{array}{c}\text { Chl } a \\
\left(\mu \mathrm{g} \mathrm{l}^{-1}\right)\end{array}$ & $\begin{array}{c}\% \\
\text { Cyano } \\
\text { bacteria }\end{array}$ & $\begin{array}{c}\text { "Bloom" } \\
\text { (intensity) }\end{array}$ & $\begin{array}{l}\text { "Bloom" } \\
\text { toxic } \\
\text { species }\end{array}$ \\
\hline$<0.998$ & $<1$ & $4 \div 5$ & $<0.9$ & $>4$ & $<4$ & $<4$ & $\div$ & no \\
\hline $0.995 \div 0.998$ & $1 \div 5$ & $3 \div 4$ & $0.9 \div 2$ & $2 \div 4$ & $4 \div 10$ & $4 \div 15$ & $\div$ & no \\
\hline $0.975 \div 0.995$ & $5 \div 8$ & $2 \div 3$ & $2 \div 10$ & $1.5 \div 2$ & $10 \div 15$ & $15 \div 20$ & I & no/yes \\
\hline $0.95 \div 0.975$ & $8 \div 10$ & $1 \div 2$ & $10 \div 20$ & $1 \div 1.5$ & $15 \div 50$ & $20 \div 50$ & II $\div$ III & yes \\
\hline$<0.95$ & $>10$ & $0 \div 1$ & $>20$ & $<1$ & $>50$ & $>\mathbf{5 0}$ & III $\div V$ & yes \\
\hline
\end{tabular}

\section{Statistical analysis}

Data analyses using package Canoco ver. 4.5 were conducted (Ter Braak and Šmilauer, 2002). Canonical ordination technique (CCA) was carried out on data for 5 environmental variables (altitude, depth, transparency acc. to Secchi - SD, phytoplankton biomass and chlorophyll a) measured at 78 lakes, total number of 
occurrences 375 , to study the role of variables related to lake's type. The data were transformed $\left(\mathrm{x}^{\prime}=\log (\mathrm{x}+1)\right)$, automatically centered and standardized by the Canoco analysis program. Monte Carlo permutation tests (number of permutations 499) and forward selection were used within CCA to detect significant $(\mathrm{p}=0.05$ probability threshold level) and independent environmental variables. Correlation coefficients (r) among the various phytoplankton metrics at studied lakes were calculated (correlation matrix).

Table 2. Mesotrophic type ,lakes" (L4, L5, L6, L7, L8, L9, L10, L14, L15, L16, L17) classification system.

\begin{tabular}{|ccccccccc}
\hline $\begin{array}{c}\text { EQR } \\
(\text { AGI })\end{array}$ & $\begin{array}{c}\text { Total } \\
\text { biomass } \\
\left(\mathrm{mg} \mathrm{I}^{-1}\right)\end{array}$ & Q & AGI & $\begin{array}{c}\text { Transp } \\
\text { arency } \\
(\mathbf{m})\end{array}$ & $\begin{array}{c}\text { Chl } a \\
\left(\mu \mathrm{I} \mathrm{I}^{-1}\right)\end{array}$ & $\begin{array}{c}\text { \% } \\
\text { Cyano } \\
\text { bacteria }\end{array}$ & $\begin{array}{c}\text { "Bloom" } \\
\text { (intensity) }\end{array}$ & $\begin{array}{c}\text { "Bloom } \\
\text { toxic } \\
\text { species }\end{array}$ \\
\hline$<0.998$ & $<1.5$ & $4 \div 5$ & $<1$ & $>4$ & $<4$ & $<4$ & $\div$ & no \\
\hline $0.994 \div 0.998$ & $1.5 \div 7$ & $3 \div 4$ & $1 \div 2.5$ & $2 \div 4$ & $4 \div 10$ & $4 \div 15$ & I & no/yes \\
\hline $0.975 \div 0.994$ & $7 \div 15$ & $2 \div 3$ & $2.5 \div 10$ & $0.5 \div 2$ & $10 \div 20$ & $15 \div 20$ & II & yes \\
\hline $0.95 \div 0.975$ & $15 \div 25$ & $1 \div 2$ & $10 \div 20$ & $0.6 \div 1$ & $20 \div 50$ & $20 \div 50$ & III & yes \\
\hline$<0.95$ & $>25$ & $0 \div 1$ & $>20$ & $<0.6$ & $>50$ & $>50$ & IV $\div$ V & yes \\
\hline
\end{tabular}

\section{Results}

\section{Lake groups division}

The relationships between environmental characteristics of the studied 78 lakes were identified using CCA (Fig. 2). The sum of all canonical eigenvalues was 0.187 . The first CCA axis explained $52 \%$ of the variance of the data. All five environmental parameters tested were significantly correlated with the lake type $(\mathrm{p}<0.01)$. Chlorophyll $a$ and biovolume, significantly correlated with the negative part of the first axis, were shown to be the parameters exerting greatest influence, explaining together $64 \%$ of the variance. Second axis explaining together with first axis $59.6 \%$ of the variance of lake types, was most strongly correlated with altitude. Studied lakes were grouped as follows. Group I at the upper right side of the CCA ordination diagram incorporated 11 alpine and mountain lakes at an altitude $>800 \mathrm{~m}$ above sea level. Alpine lakes over $2240 \mathrm{~m}$ (I.1, i.e. No 69, 70, 75 and 78) formed a distinguished subgroup among them (I.1). The second group (II) included 27 deep semi mountain and lowland lakes, at less than $800 \mathrm{~m}$ a.s.l. and over $8.5 \mathrm{~m}$ maximal depth. Axis 2 separated the above two groups oligotrophic lakes at the right side of the plot from Group III, combining 38 mesotrophic small and middle sized lowland lakes at an altitude $<200 \mathrm{~m}$ a.s.l. and size up to $10 \mathrm{~km}^{2}$. Two lakes from national type L10 Black Sea hyperhaline coastal lakes, included at Group III: Pomorijsko (No 48) and Atanasovsko lakes (No 49), differ strongly in salinity $(>42 \%)$. Thus they were discussed as separate Group IV. 
10

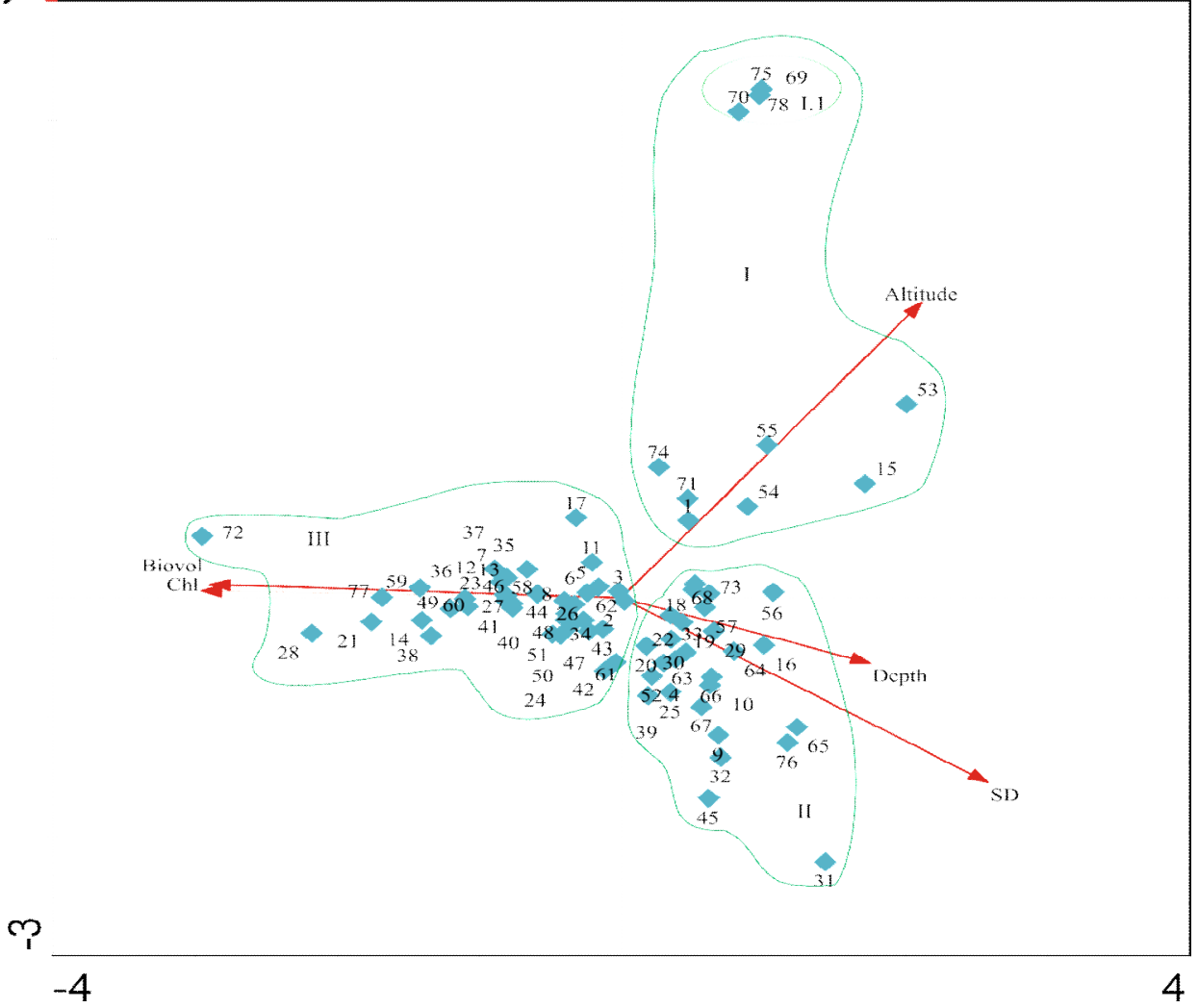

Figure 2. CCA ordination diagram of 5 environmental variables measured at 78 studied lakes from all lake types. Explanations: Arrows - relevant variable value, starts from the origin with average values and extends toward higher values $(\mathrm{Chl}=$ Chlorophyll $a$; Biovol $=$ Biovolume, $S D=$ Transparency according to Secchi); diamonds - relevant sampling site according Fig. 1;

lake groups: I.1 - Alpine lakes; I-Mountain lakes; II - Deep semi mountain and lowland lakes; III Small and middle sized lowland lakes

\section{Physico-chemical water quality analysis}

Data for physico-chemical parameters of lake water were presented at Table 3. Average temperature and $\mathrm{pH}$ increased in comformity from Group I (Alpine and Mountain lakes) to Group III and IV (lowlands lakes), while transparency decreased. Electrical conductivity and salinity were highest at lakes from Group IV due to their transitional character.

Table 3. Range of environmental variables for the different "lake" groups in 2009. Legend: Groups according Fig. 2; T, Temperature; DO, Dissolved oxygen; OS, Oxygen saturation; SD, Secchi depth; Cond, Conductivity; $\mathrm{N}-\mathrm{NH} 4+$, Ammonium nitrogen; $\mathrm{N}-\mathrm{NO} 2-$, Nitrite nitrogen; $\mathrm{N}-\mathrm{NO} 3-$, Nitrate nitrogen; SRP, Soluble reactive phosphorus; TP, Total phosphorus; TN, Total nitrogen; Chl a, Chlorophyll a; S, Salinity; Ls, Lake size.

\begin{tabular}{lllllllll}
\hline & \multicolumn{2}{c}{$\begin{array}{c}\text { Group I } \\
\mathrm{n}=11\end{array}$} & \multicolumn{2}{c}{$\begin{array}{c}\text { Group II } \\
\mathrm{n}=27\end{array}$} & \multicolumn{2}{c}{$\begin{array}{c}\text { Group III } \\
\mathrm{n}=38\end{array}$} & \multicolumn{2}{c}{$\begin{array}{c}\text { Group IV } \\
\mathrm{n}=2\end{array}$} \\
\cline { 2 - 8 } & $\begin{array}{l}\text { Interva } \\
1\end{array}$ & Mean & Interval & Mean & Interval & Mean & Interval & Mean \\
& & & & & & & \\
\hline $\mathbf{T}^{\mathbf{0}} \mathbf{C}$ & $3.0-$ & $\mathbf{1 3 . 5}$ & $10.8-28$ & $\mathbf{1 9 . 0}$ & $9.2-29.2$ & $\mathbf{1 9 . 6}$ & $16.9-18.4$ & $\mathbf{1 7 . 7}$ \\
& 20.9 & & & & & & & \\
\hline $\begin{array}{l}\text { DO } \\
\left(\mathbf{m g ~ l}^{\mathbf{1}}\right)\end{array}$ & $5.8-8.0$ & $\mathbf{7 . 4}$ & $6.4-9.8$ & $\mathbf{7 . 9}$ & $1.1-18.4$ & $\mathbf{7 . 9}$ & $4.9-5.8$ & $\mathbf{5 . 4}$ \\
\hline
\end{tabular}




\begin{tabular}{|c|c|c|c|c|c|c|c|c|}
\hline OS (\%) & $65-94$ & 82.5 & $66-109$ & 85.4 & $\begin{array}{l}28.9- \\
214\end{array}$ & 85.6 & $52.3-58.6$ & 55.5 \\
\hline SD (m) & $\begin{array}{l}1.6- \\
16.0\end{array}$ & 7.10 & $1.1-7.5$ & 3.3 & $0.3-2.3$ & 1.0 & $0.25-0.7$ & 0.5 \\
\hline pH & $7.2-8.4$ & 7.8 & $6.7-9.0$ & 8.2 & $7.7-9.6$ & 8.6 & $8.4-8.9$ & 8.7 \\
\hline $\begin{array}{l}\text { Cond } \\
\left.(\mu \mathrm{S} \mathrm{cm})^{-1}\right)\end{array}$ & $\begin{array}{l}9.7- \\
308 \\
\end{array}$ & 86.2 & $22-536$ & 244 & $\begin{array}{l}176- \\
1864\end{array}$ & 591 & $\begin{array}{l}64000- \\
105003\end{array}$ & 84502 \\
\hline $\begin{array}{l}\mathrm{N}-\mathrm{NH}_{4}^{+} \\
\left(\mathrm{mg} \mathrm{l}^{-1}\right)\end{array}$ & $\begin{array}{l}0.01- \\
0.22\end{array}$ & 0.039 & $\begin{array}{l}0.006- \\
0.09\end{array}$ & 0.026 & $\begin{array}{l}0.008- \\
1.55\end{array}$ & 0.15 & $\begin{array}{l}0.17- \\
0.065\end{array}$ & 0.118 \\
\hline $\begin{array}{l}\mathrm{N}-\mathrm{NO}_{2}^{-} \\
\left(\mathrm{mg} \mathrm{l}^{-1}\right)\end{array}$ & $\begin{array}{l}0.002- \\
0.005\end{array}$ & 0.003 & $\begin{array}{l}0.002- \\
0.04\end{array}$ & 0.006 & $\begin{array}{l}0.002- \\
0.067\end{array}$ & 0.02 & $\begin{array}{l}0.007- \\
0.025\end{array}$ & 0.016 \\
\hline $\begin{array}{l}\mathrm{N}-\mathrm{NO}_{3}^{-} \\
\left(\mathrm{mg} \mathrm{l}^{-1}\right)\end{array}$ & $\begin{array}{l}0.035- \\
0.07\end{array}$ & 0.157 & $\begin{array}{l}0.08- \\
0.45\end{array}$ & 0.212 & $\begin{array}{l}0.066- \\
0.80\end{array}$ & 0.27 & $0.2-0.6$ & 0.4 \\
\hline $\begin{array}{l}\text { SRP } \\
\left(\mathrm{mg} \mathrm{l}^{-1}\right)\end{array}$ & $\begin{array}{l}0.01- \\
0.083\end{array}$ & 0.027 & $\begin{array}{l}0.005- \\
0.034\end{array}$ & 0.012 & $\begin{array}{l}0.001- \\
0.317\end{array}$ & 0.04 & $\begin{array}{l}0.023- \\
0.059\end{array}$ & 0.041 \\
\hline $\begin{array}{l}\text { TP } \\
\left(\mathrm{mg} \mathrm{l}^{-1}\right)\end{array}$ & $\begin{array}{l}0.002- \\
0.263 \\
\end{array}$ & 0.075 & $\begin{array}{l}0.008- \\
0.091\end{array}$ & 0.024 & $\begin{array}{l}0.010- \\
0.364\end{array}$ & 0.107 & $\begin{array}{l}0.07- \\
0.096\end{array}$ & 0.083 \\
\hline $\begin{array}{l}\text { TN } \\
\left(\mathrm{mg} \mathrm{l}^{-1}\right)\end{array}$ & $\begin{array}{l}0.50- \\
0.50 \\
\end{array}$ & 0.50 & $0.4-1.9$ & 0.762 & $\begin{array}{l}0.378- \\
4.10 \\
\end{array}$ & 1.128 & $1.9-3$ & 2.5 \\
\hline $\begin{array}{l}\text { Chl } a \\
\left(\mu g ~^{-1}\right)\end{array}$ & $\begin{array}{l}<0.2- \\
6.5 \\
\end{array}$ & 2.65 & $\begin{array}{l}<0.2- \\
14.5 \\
\end{array}$ & 2.98 & $\begin{array}{l}0.2- \\
119.3 \\
\end{array}$ & 24.68 & $2.61-31.2$ & 16.91 \\
\hline S (\%o) & $<0.5$ & & $<0.5$ & & $<0.5$ & & $42.7-63.4$ & 53.1 \\
\hline $\begin{array}{l}\mathbf{L s} \\
\left(\mathrm{km}^{2}\right)\end{array}$ & $\begin{array}{l}0.02- \\
22.08\end{array}$ & 3.11 & $\begin{array}{l}0.03- \\
30.0\end{array}$ & 7.68 & $\begin{array}{l}0.04- \\
13.0\end{array}$ & 2.42 & $9.5-16.9$ & 13.2 \\
\hline
\end{tabular}

\section{Assemblage index (Q)}

Functional groups

A total of 27 phytoplankton FGs were identified on the basis of selected 19 lakes from the dataset (Table 4 and Table 5).

Two lakes were included in the Lake Subgroup I.1 - Alpine lakes (Table 4). Chernoto and Bezbog Lake are small sized $\left(<0.15 \mathrm{~km}^{2}\right)$ glacial lakes located at altitude above $2240 \mathrm{~m}$. They were characterized by high transparency and ultra oligotrophic conditions. Six FGs were recoreded: $\mathbf{L}_{\mathbf{0}}, \mathbf{Y}, \mathbf{F}, \mathbf{M P}, \mathbf{E}$ and $\mathbf{X 3}$ (Tables 4, 5). FG $\mathbf{L}_{\mathbf{0}}$ dominated as $2 / 3$ from biomass was represented by unicellular dinoflagellates with large size: Peridinium cinctum - 77.2\% (Chernoto Lake) and Gymnodinium palustre - 73.7\% (Bezbog Lake). Colonial mucilaginous chlorococcocales from FG F: Oocystis lacustris - 5.3\% (Chernoto Lake) and Radiococcus nimbatus - 5.2\% (Bezbog Lake) were also dominant species with biomass $>5 \%$.

Three reservoirs were selected as representative for the Lake Group I - Mountain lakes: Belmeken, Beli Iskar and Toshkov chark reservoirs. They are small to middle sized deep reservoirs, situated at above $1400 \mathrm{~m}$ a.s.l. Transparency over $3.5 \mathrm{~m}$ and oligotrophic conditions were assessed. Besides the above six FGs at alpine lakes, another five groups were determined: $\mathbf{B}, \mathbf{C}, \mathbf{G}, \mathbf{N}$ and $\mathbf{W 2}$ (Table 4 and Table 5). The following groups were dominant in biomass: $\mathbf{L}_{\mathbf{0}}, \mathbf{E}, \mathbf{N}$ and $\mathbf{C}$ (Table 4). Dinoflagellates from $\mathbf{L}_{\mathbf{0}}$ : Peridinium cinctum (53.1\%), Gymnodinium sp. (31.6\%) and Gymnodinium sp. $(59.2 \%)$ were first dominant taxa at Belmeken and Beli Iskar. Second dominants were large filamentous desmid Spondylosium planum (8.9\%) from $\mathbf{N}$ at Belmeken Reservoir, 
and colonial chrysophycean Stichogloea doederleinii (22.4\%) from X2 at Beli Iskar Reservoir. Flagellate chrysophyceans from FG E Dinobryon divergens (27.8\%), Mallomonas sp. (22.2\%), Mallomonas caudata (11.1\%) and colonial diatom from C Asterionella formosa (11.1\%) were dominant taxa at Toshkov chark Reservoir.

Lake Group II - Deep semi mountain and lowland lakes included 8 reservoires, located between 118 (Ivaylovgrad Reservoir) and $529 \mathrm{~m}$ a.s.l. (Vacha Reservoir) and characterized by presence of thermocline in summer and profundal zone. A total of 21 FGs were designated - in addition to 11 groups at Mountain lakes, 10 new groups were recorded: J, X1, P, W1, D, H1, X2, Z, U, S1 (Tables 4, 5). Dominant position of $\mathbf{L}_{\mathbf{0}}, \mathbf{E}$, $\mathbf{N}$ and $\mathbf{C}$ at Mountain lakes was replaced by $\mathbf{F}, \mathbf{P}, \mathbf{X 3}, \mathbf{B}, \mathbf{H 1}$ and $\mathbf{J}$. Taxa from group $\mathbf{F}$ were first dominant at some of reservoirs (Studen kladenets, Kardzhali and Ivaylovgrad) (Table 4). Group F was represented from colonial mucilaginous chlorococcocales Oocystis lacustris $(9.5 \%)$ and Sphaerocystis planctonica $(9.5 \%)$ at Kardzhali Reservoir, from Oocystis ecballocystiformis (26.7\%) at Studen kladenets Reservoir and Oocystis solitaria (67.8\%) and Planctococcus sphaerocystiformis (7.9\%) at Ivaylovgrad Reservoir. At other water bodies, first dominant were species from group P, e.g. at Krichim Reservoir colonial diatom Fragilaria crotonensis, monodominant $(85.5 \%)$, colonial diatom Aulacoseira granulata $(67.8 \%)$ at Borovista Reservoir, unicells desmids Staurastrum teliferum (23.7\%) and Staurastrum gracile $(20.5 \%)$ at Devets Reservoir. Only at Srechenska bara Reservoir species from FG $\mathbf{L}_{\mathbf{0}}$ was first dominant - unicellular dinoflagellate with large size Gymnodinium sp. (61.4\%). Vacha Reservoir was an isolated case in the group Deep semi mountain and lowland lakes. Regardless of its highest altitude, first and second dominant in the total biomass were species from FGs $\mathbf{J}$ - Crucigeniella rectangularis (36.7\%) and $\mathbf{H 1}$ - Aphanizomenon flos-aquae (33.6\%). Overall assessment revealed worst ecological status among other water bodies in the group.

Third lake group Small and middle sized lowland lakes covered one swamp and 4 reservoirs. Durankulak swamp belongs to national lake type L7 (Black Sea freshwater coastal lakes, salinity $<0.5 \%$ ), reservoirs to L12 (Eleshniza and Ovcharitsa) and L16 (Poroj and Acheloy). Their altitude ranged between $0 \mathrm{~m}$ (Durankulak swamp) and 142 $\mathrm{m}$ (Acheloy Reservoir). Except for Durankulak swamp (Depth max $-1.70 \mathrm{~m}$ ), which is shallow polymictic, the rest water bodies have average depth (Depth max $_{1}: 11.3 \div 22.3 \mathrm{~m}$ ), and thermocline in the summer season. All lakes were in mesotrophic to eutrophic conditions. Twenty-five FGs were registered (Tables 4, 5). Additionally to the 21 FGs for Lake Group II, $\mathbf{L}_{\mathbf{M}}, \mathbf{X}_{\mathbf{P h}}, \mathbf{K}, \mathbf{T}, \mathbf{M}, \mathbf{S 2}$ were recoreded at Lake Group III. At lowland lakes no particular dominants were established, possibly due to the particular extent of athropogenic pressure. Almost half of the biomass was formed by group $\mathbf{J}(44.2 \%)$, represented from coenobial chlorococcocales Crucigeniella crucifera, Crucigenia quadrata, Pediastrum boryanum, Scenedesmus disciformis and $S$. opoliensis at Durankulak swamp. Second dominant was colonial cyanobacteria Microcystis aeruginosa (9.0\%) - group M. At Eleshniza Reservoir with equal share were picoplanctonic green algae Stichococcus minutissimum (12.5\%) from X3, colonial chlorococcocales Coelastrum polychordum (12.5\%) from $\mathbf{J}$, and Phacotus coccifer $(12.5 \%)$ from $\mathbf{X}_{\mathbf{p h}}$. First dominant at Poroj Reservoir was from X2 (Chlamydomonas sp. - 33.3\%), second from $\mathbf{B}$ (Cyclotella $\mathrm{sp} .-13.7 \%$ ). Ceratium furcoides from $\mathbf{L}_{\mathbf{M}}$ was first dominant (38.4\%) at Ovcharitsa Reservoir together with Microcystis aeruginosa, second from P (Aulacoseira granulata - 37.5\%). Biomass at Acheloy Reservoir was formed almost only from eutrophic species cyanobacteria, belonging to S1 (Limnothrix 
redekei - 64.3\%, Pseudanabaena limnetica - 19.7\%) and H1 (Aphanizomenon flosaquae - 5.7\%).

From Lake Group IV (Black Sea hyperhaline coastal lakes) was studied Atanasovsko Lake (national type L10 Black Sea hyperhaline coastal lakes, Transitional Waters), with salinity $>40 \%$. Only 4 FGs were registered: X2, L $\mathbf{0}$, D and MP (Tables 4, 5). About $90 \%$ of biomass was formed by group $\mathbf{X} \mathbf{2}$, to which belonged flagellates green algae Provasoliella ovata (52.4\%) and Pyramimonas tetrarhynchus (35.5\%).

Table 4. Main phytoplankton species ( $>5 \%$ of the total biomass) with their taxonomic and functional groups. In parenthesis is given the total number of the studied lakes.

\begin{tabular}{|c|c|c|c|c|c|}
\hline Name of the Locality & $\begin{array}{l}\text { Lake } \\
\text { Type }\end{array}$ & Phytoplankton Species & $\begin{array}{l}\text { Relative } \\
\text { biomass } \\
(\%)\end{array}$ & Taxonomic Group & $\begin{array}{l}\text { Functional } \\
\text { Group }\end{array}$ \\
\hline \multicolumn{6}{|c|}{ Lake Subgroup I.1- Alp ine lakes (n=4) } \\
\hline \multirow[t]{3}{*}{ Chernoto Lake } & $\mathrm{L} 1$ & Peridinium cinctum (O.F.M.) Ehr. & 77.2 & Dinophyceae & $\mathrm{L}_{0}$ \\
\hline & & Cryptomonas sp. & 7.0 & Cryptophyceae & $\mathrm{Y}$ \\
\hline & & Oocystis lacustris Chod. & 5.3 & Chlorophyceae & $\mathrm{F}$ \\
\hline \multirow[t]{3}{*}{ Bezbog Lake } & L1 & Gymnodinium palustre Schilling & 73.7 & Dinophyceae & $\mathrm{L}_{0}$ \\
\hline & & Diatoma mesodon (Ehr.) Kütz. & 7.4 & Bacillariophyceae & MP \\
\hline & & Radiococcus nimbatus (De-Wild.) Schmidle & 5.2 & Chlorophyceae & $\mathrm{F}$ \\
\hline \multicolumn{6}{|c|}{ Lake Group I - Mou ntain „lakes” (n=7) } \\
\hline \multirow[t]{4}{*}{ Belmeken Res. } & L13 & Peridinium cinctum (O.F.M.) Ehr. & 53.1 & Dinophyceae & $\mathrm{L}_{0}$ \\
\hline & & Gymnodinium sp. & 31.6 & Dinophyceae & $\mathrm{L}_{0}$ \\
\hline & & Aphanothece clathrata W. et G.S. West & 5.1 & Cyanoprokaryota & $\mathrm{L}_{0}$ \\
\hline & & Spondylosium planum (Wolle) W. et G.S. West & 8.9 & Zygnemaphyceae & $\mathrm{N}$ \\
\hline \multirow[t]{2}{*}{ Beli Iskar Res. } & L1 & Gymnodinium sp. & 59.2 & Dinophyceae & $\mathrm{L}_{0}$ \\
\hline & & Stichogloea doederleinii (Schmidle) Wille & 22.4 & Chrysophyceae & $\mathrm{X} 2$ \\
\hline \multirow[t]{9}{*}{ Toshkov chark Res. } & L3 & Dinobryon divergens $\operatorname{Imh}$. & 27.8 & Chrysophyceae & $\mathrm{E}$ \\
\hline & & Mallomonas sp. & 22.2 & Chrysophyceae & $\mathrm{E}$ \\
\hline & & Mallomonas caudata Iwanoff & 11.1 & Chrysophyceae & $\mathrm{E}$ \\
\hline & & $\begin{array}{l}\text { Asterionella formos a var. gracillima (Hanztsch in } \\
\text { Rabenh.) Grun. }\end{array}$ & 11.1 & Bacillariophyceae & $\mathrm{C}$ \\
\hline & & Aphanocapsa delicatissima W. et G.S. West & 5.6 & Cyanoprokaryota & $\mathrm{L}_{0}$ \\
\hline & & Sphaerocystis planctonica (Korshikov) Bourrelly & 5.6 & Chlorophyceae & $\mathrm{F}$ \\
\hline & & Cyclotella sp. & 5.6 & Bacillariophyceae & $\mathrm{B}$ \\
\hline & & Pandorina morum (O.F. Müller) Bory & 5.6 & Chlorophyceae & $\mathrm{G}$ \\
\hline & & Trachelomonas volvocina Ehr. & 5.6 & Euglenophyceae & W2 \\
\hline \multicolumn{6}{|c|}{ Lake Group II - Deep semi mountain and lowland "lakes" (n=27) } \\
\hline \multirow[t]{4}{*}{ Srechenska bara Res. } & $\mathrm{L} 2$ & Gymnodinium sp. & 61.4 & Dinophyceae & $\mathrm{L}_{0}$ \\
\hline & & Chrysococcus punctiformis $\mathrm{P}$ asch. & 22.8 & Chrysophyceae & $\mathrm{X} 3$ \\
\hline & & Cyclotella sp. & 7.5 & Bacillariophyceae & B \\
\hline & & Aulacoseira sp. & 5.0 & Bacillariophyceae & $\mathrm{P}$ \\
\hline \multirow[t]{8}{*}{ Kardzhali Res. } & $\mathrm{L} 11$ & Cyclotella $\mathrm{sp}$. & 16.7 & Bacillariophyceae & $\mathrm{B}$ \\
\hline & & Oocystis lacustris Chod. & 9.5 & Chlorophyceae & $\mathrm{F}$ \\
\hline & & Sphaerocystis planctonica (Korshikov) Bourrelly & 9.5 & Chlorophyceae & $\mathrm{F}$ \\
\hline & & Aphanothece clathrata W. et G.S. West & 9.5 & Cyanoprokaryota & $\mathrm{L}_{0}$ \\
\hline & & Uroglena articulata Korshikov & 9.5 & Chrysophyceae & $\mathrm{U}$ \\
\hline & & Schroederia spiralis (Printz) Korš. & 7.1 & Chlorophyceae & $\mathrm{X} 3$ \\
\hline & & Chroomonas acuta Uterm. & 7.1 & Cryptophyceae & $\mathrm{X} 2$ \\
\hline & & Euglena sp. & 9.5 & Euglenophyceae & W1 \\
\hline \multirow[t]{4}{*}{ Studen kladenets Res. } & $\mathrm{L} 11$ & Oocystis ecballocystiformis lyengar & 26.7 & Chlorophyceae & $\mathrm{F}$ \\
\hline & & Tetrachlor ella alternans (G.M. Smith) Korš. & 25.0 & Chlorophyceae & $\mathrm{X} 3$ \\
\hline & & Rhopalodia gibba (Ehr.) O. Müll. & 17.2 & Bacillariophyceae & MP \\
\hline & & Cymatopleura solea (Brébis son) W.Smith & 14.6 & Bacillariophyceae & MP \\
\hline \multirow[t]{3}{*}{ Ivaylovgrad Res. } & L11 & Oocystis solitaria Wittr. in Wittr. \& Nordst. & 67.8 & Chlorophyceae & $\mathrm{F}$ \\
\hline & & Coelastrum polychordum (Korš.) Hind. & 10.9 & Chlorophyceae & $\mathrm{J}$ \\
\hline & & Planctococcus sphaerocystiformis Korš. & 7.9 & Chlorophyceae & $\mathrm{F}$ \\
\hline \multirow[t]{6}{*}{ Devets Res. } & L16 & Staurastrum teliferum Ralfs & 23.7 & Zygnemaphyceae & $\mathrm{P}$ \\
\hline & & Staurastrum gracile Ralfs & 20.5 & Zygnemaphyceae & $\mathrm{P}$ \\
\hline & & Euastrum sp. & 20.5 & Zygnemaphyceae & $\mathrm{N}$ \\
\hline & & Chroococcus sp. & 12.7 & Cyanoprokaryota & $\mathrm{L}_{0}$ \\
\hline & & Amphora sp. & 7.0 & Bacillariophyceae & MP \\
\hline & & Chrysococcus rufescens Klebs & 5.0 & Chrysophyceae & $\mathrm{X} 3$ \\
\hline Krichim Res. & L11 & Fragilaria crotonensis Kitt. & 85.5 & Bacillariophyceae & $\mathrm{P}$ \\
\hline \multirow[t]{3}{*}{ Borovista Res. } & L11 & Aulacoseira granulata (Ehrenb.) Sim. & 67.8 & Bacillariophyceae & $\mathrm{P}$ \\
\hline & & Radiococcus planctonicus Lund & 7.3 & Chlorophyceae & $\mathrm{F}$ \\
\hline & & Volvox aureus Ehrenberg & 5.5 & Chlorophyceae & $\mathrm{G}$ \\
\hline \multirow[t]{4}{*}{ Vacha Res. } & L11 & Crucigeniella rectangularis (Näg.) Kom. & 36.7 & Chlorophyceae & $\mathrm{J}$ \\
\hline & & Aphanizomenon flos-aquae (L.) Ralfs & 33.6 & Cyanoprokaryota & $\mathrm{H} 1$ \\
\hline & & Sphaerocystis planctonica (Korshikov) Bourrelly & 11.4 & Chlorophyceae & $\mathrm{F}$ \\
\hline & & Radiococcus planctonicus Lund & 9.1 & Chlorophyceae & $\mathrm{F}$ \\
\hline
\end{tabular}




\begin{tabular}{|c|c|c|c|c|c|}
\hline \multicolumn{6}{|c|}{ Lake Group II I - Small and middle sized lowland “lakes" (n=38) } \\
\hline \multirow[t]{6}{*}{ Eleshnitsa Res. } & L12 & Coelastrum polychordum (Korš.) Hind. & 12.5 & Chlorophyceae & $\mathrm{J}$ \\
\hline & & Stichococcus minutissimus Skuja & 12.5 & Chlorophyceae & $\mathrm{X} 3$ \\
\hline & & Phacotus coccifer Korschikoff & 12.5 & Chlorophyceae & $\mathrm{X}_{\mathrm{Ph}}$ \\
\hline & & Staurastrum sp. & 10.4 & Zygnemaphyceae & $P$ \\
\hline & & Ceratium hirundinella (O.F.Müller) Dujardin & 6.3 & Dinophyceae & $\mathrm{L}_{0}$ \\
\hline & & Cosmarium sp. & 6.3 & Zygnemaphyceae & $\mathrm{N}$ \\
\hline \multirow[t]{6}{*}{ Poroj Res. } & L16 & Chlamydomonas sp. & 33.3 & Chlorophyceae & $\mathrm{X} 2$ \\
\hline & & Cyclotella sp. & 13.7 & Chlorophyceae & B \\
\hline & & Gymnodinium sp. & 10.2 & Dinophyceae & $\mathrm{L}_{0}$ \\
\hline & & Golenkinia radiata Chod. & 6.8 & Chlorophyceae & $\mathrm{J}$ \\
\hline & & Euglena limnophila Lemm. & 6.8 & Euglenophyceae & W 1 \\
\hline & & Aulacoseira granulata (Ehrenb.) Sim. & 5.0 & Bacillariophyceae & $\mathrm{P}$ \\
\hline \multirow[t]{3}{*}{ Ovcharitsa Res. } & L12 & Ceratium furcoides (Levander) Langhans & 38.4 & Dinophyceae & $\mathrm{L}_{\mathrm{M}}$ \\
\hline & & Aulacoseira granulata (Ehrenb.) Sim. & 37.5 & Bacillariophyceae & $\mathrm{P}$ \\
\hline & & Carteria multifilis (Fresenius) Dill & 15.1 & Chlorophyceae & G \\
\hline \multirow[t]{7}{*}{ Durankulak swamp } & L7 & Crucigeniella crucifera (Wolle) Kom. & 13.4 & Chlorophyceae & $\mathrm{J}$ \\
\hline & & Crucigenia quadrata Morr. & 11.7 & Chlorophyceae & $\mathrm{J}$ \\
\hline & & Scenedesmus opoliensis P.G. Richt. & 7.9 & Chlorophyceae & $\mathrm{J}$ \\
\hline & & Pediastrum boryanum (Turpin) Meneghini & 6.2 & Chlorophyceae & $\mathrm{J}$ \\
\hline & & Scenedesmus disciformis (Chod.) Fott \& Kom. & 5.0 & Chlorophyceae & $\mathrm{J}$ \\
\hline & & Microcystis a eruginosa (Kütz.) Kütz. & 8.9 & Cyanoprokaryota & M \\
\hline & & Anabaena spiroides Kleb. & 5.0 & Cyanoprokaryota & H1 \\
\hline \multirow[t]{3}{*}{ Acheloy Res. } & L16 & Limnothrix redekei (Van Goor) Meffert van Goor & 64.3 & Cyanoprokaryota & S1 \\
\hline & & Pseudanabaena limnetica (Lemmermann) Komárek & 19.7 & Cyanoprokaryota & S1 \\
\hline & & Aphanizomenon flos-aquae (L.) Ralfs & 5.7 & Cyanoprokaryota & $\mathrm{H} 1$ \\
\hline \multicolumn{6}{|c|}{ Lake Group IV - Black Sea hyperhaline coastal lakes $(n=2)$} \\
\hline \multirow[t]{3}{*}{ Atanasovsko Lake } & L10 & Provasoliella ovata (Jac.) A.R. Loebl. & 52.4 & $\begin{array}{l}\text { Chlamydophyceae } \\
\text { (Chlorophyta) }\end{array}$ & $\mathrm{X} 2$ \\
\hline & & Pyramimonas tetrarhynchus Schmarda & 35.5 & $\begin{array}{l}\text { Prasinophyceae } \\
\text { (Chlorophyta) }\end{array}$ & $\mathrm{X} 2$ \\
\hline & & Gymnodinium splendens Lebour & 5.4 & Dinophyceae & $\mathrm{L}_{0}$ \\
\hline
\end{tabular}

\section{Factor numbers $(F)$}

Determination of $\mathrm{F}$ was based on expert knowledge, existing literature data and our background with $\mathrm{F}$ calibration at lakes under reference conditions, as well as at lakes with assessed indisputable overall status. The F values per lake groups and types were presented at Table 5, as well as literature data. It has to be noticed that Q was calculated on a set of 19 lakes from the total database of 78 water bodies, thus $\mathrm{F}$ was valid only for national types L1, L2, L3, L7, L10, L11, L12, L13, L16 (Table 5).

Table 5. Factor number $(F)$ to the phytoplankton functional groups per lake groups and types. *Functional groups covering dominant species (biomass $>5 \%$ from the total biomass). L: Lake type. References: A - Padisák et al. (2006); B - Crossetti and Bicudo (2008); C Becker et al. (2009); D - Becker et al. (2010).

\begin{tabular}{|c|c|c|c|c|c|}
\hline \multirow[b]{2}{*}{$\begin{array}{l}\text { Functional } \\
\text { groups }\end{array}$} & \multicolumn{5}{|c|}{$\begin{array}{l}\text { Lake Groups } \\
\end{array}$} \\
\hline & $\begin{array}{l}\text { I.1 } \\
\text { Alpine } \\
\text { lakes } \\
\text { L1 }\end{array}$ & $\begin{array}{c}\text { I } \\
\text { Mountain } \\
\text { lakes } \\
\text { L3, L13 }\end{array}$ & $\begin{array}{c}\text { II } \\
\text { Deep semi } \\
\text { mountain } \\
\text { and } \\
\text { lowland } \\
\text { lakes } \\
\text { L2, L11 }\end{array}$ & $\begin{array}{c}\text { III } \\
\text { Small and middle } \\
\text { sized lowland lakes } \\
\text { L7, L12, L16 }\end{array}$ & $\begin{array}{c}\text { IV } \\
\text { Black Sea } \\
\text { hyperhaline } \\
\text { coastal lakes } \\
\text { L10 }\end{array}$ \\
\hline $\mathbf{B}$ & & $* 3$ & $* 5$ & $\begin{array}{l}* 5 \\
\text { A } 5\end{array}$ & \\
\hline $\bar{C}$ & & $* 2$ & $\begin{array}{c}3 \\
\text { C } 2 \\
\text { D } 3\end{array}$ & $\begin{array}{c}5 \\
\text { A } 5\end{array}$ & \\
\hline $\mathbf{D}$ & & & $\begin{array}{c}2 \\
\text { C } 2 \\
\text { D } 2\end{array}$ & $\begin{array}{l}2,5 \\
\text { A } 3 \\
\text { B } 2\end{array}$ & 2,5 \\
\hline
\end{tabular}


Belkinova et al.: Phytoplankton based assessment of ecological status of Bulgarian lakes and comparison of metrics within the Water Framework Directive -94 -

\begin{tabular}{|c|c|c|c|c|c|}
\hline $\mathbf{N}$ & & $* 5$ & $\begin{array}{c}* 5 \\
\text { C } 5 \\
\text { D } 5 \\
\end{array}$ & $\begin{array}{l}* 5 \\
\text { A } 5 \\
\text { B } 5 \\
\end{array}$ & \\
\hline $\mathbf{P}$ & & & $\begin{array}{c}* 3 \\
\text { C } 2 \\
\text { D } 0\end{array}$ & $\begin{array}{c}* 5 \\
\text { A } 5 \\
\text { B } 2\end{array}$ & \\
\hline MP & $* 5$ & 5 & $\begin{array}{c}* 3 \\
\text { D } 1\end{array}$ & $\begin{array}{l}* 3 \\
\text { A } 3 \\
\end{array}$ & 3 \\
\hline $\mathbf{T}$ & & & & $\begin{array}{c}5 \\
\text { A } 5 \\
\text { B } 5 \\
\end{array}$ & \\
\hline S1 & & & $\begin{array}{c}0 \\
\mathrm{C} 0 \\
\mathrm{D} 0\end{array}$ & $\begin{array}{l}* * 0 \\
\text { A } 0 \\
\text { B } 0 \\
\end{array}$ & \\
\hline $\mathbf{S 2}$ & & & & $\begin{array}{c}0 \\
\text { A } 2 \\
\end{array}$ & \\
\hline $\mathbf{Z}$ & & & 5 & & \\
\hline $\mathrm{X3}$ & 5 & 5 & $\begin{array}{c}* 5 \\
\text { D } 5\end{array}$ & $\begin{array}{c}* 5 \\
\text { A } 4 \\
\text { B } 4 \\
\end{array}$ & \\
\hline $\mathrm{X} 2$ & & & $\begin{array}{c}* 5 \\
\text { C } 5\end{array}$ & $\begin{array}{c}* 3 \\
\text { A 3,5 } \\
\text { B 5 }\end{array}$ & $* 3$ \\
\hline $\mathrm{X} 1$ & & & $\begin{array}{c}2,5 \\
\text { C } 2 \\
\text { D 3,5 } \\
\end{array}$ & $\begin{array}{l}2,5 \\
\text { A 3 } \\
\text { B 5 } \\
\end{array}$ & \\
\hline $\mathbf{X}_{\mathbf{P h}}$ & & & & $\begin{array}{l}* 3,5 \\
\text { A } 3,5\end{array}$ & \\
\hline $\mathbf{E}$ & 5 & $* 5$ & $\begin{array}{c}5 \\
\text { C } 5 \\
\text { D } 5 \\
\end{array}$ & $\begin{array}{c}5 \\
\text { A } 5 \\
\text { B } 5 \\
\end{array}$ & \\
\hline $\mathbf{Y}$ & $* 3$ & 3 & $\begin{array}{c}3 \\
\text { C 3 } \\
\text { D 3 } \\
\end{array}$ & $\begin{array}{c}3,5 \\
\text { A 3.5 } \\
\text { B 3 } \\
\end{array}$ & \\
\hline $\mathbf{F}$ & $* 2$ & $* 2$ & $\begin{array}{c}* 3 \\
\text { C } 2 \\
\text { D } 2 \\
\end{array}$ & $\begin{array}{l}* 5 \\
\text { A } 3 \\
\text { B } 5 \\
\end{array}$ & \\
\hline $\mathbf{G}$ & & $* 2$ & $* 3$ & $\begin{array}{c}* 3 \\
\text { A } 4\end{array}$ & \\
\hline $\mathbf{J}$ & & & $\begin{array}{c}* 2 \\
\text { C } 2 \\
\text { D } 2 \\
\end{array}$ & $\begin{array}{l}* 3 \\
\text { A } 3 \\
\text { B } 5 \\
\end{array}$ & \\
\hline $\mathbf{K}$ & & & & $\begin{array}{c}3 \\
\text { A } 2 \\
\text { B 3 } \\
\end{array}$ & \\
\hline H1 & & & $\begin{array}{c}* * 0 \\
\text { C } 0 \\
\text { D } 0 \\
\end{array}$ & $\begin{array}{c}* 1 \\
\text { A } 1 \\
\text { B } 1 \\
\end{array}$ & \\
\hline $\mathbf{U}$ & & & $* 4$ & & \\
\hline $\mathbf{L}_{0}$ & $* 5$ & $* 5$ & $\begin{array}{c}* 5 \\
\text { C } 5 \\
\text { D } 4 \\
\end{array}$ & $\begin{array}{l}* 5 \\
\text { A } 5 \\
\text { B } 5 \\
\end{array}$ & $* 5$ \\
\hline
\end{tabular}

APPLIED ECOLOGY AND ENVIRONMENTAL RESEARCH 12(1): 83-103. http://www.aloki.hu • ISSN 15891623 (Print) • ISSN 17850037 (Online) (c) 2014, ALÖKI Kft., Budapest, Hungary 


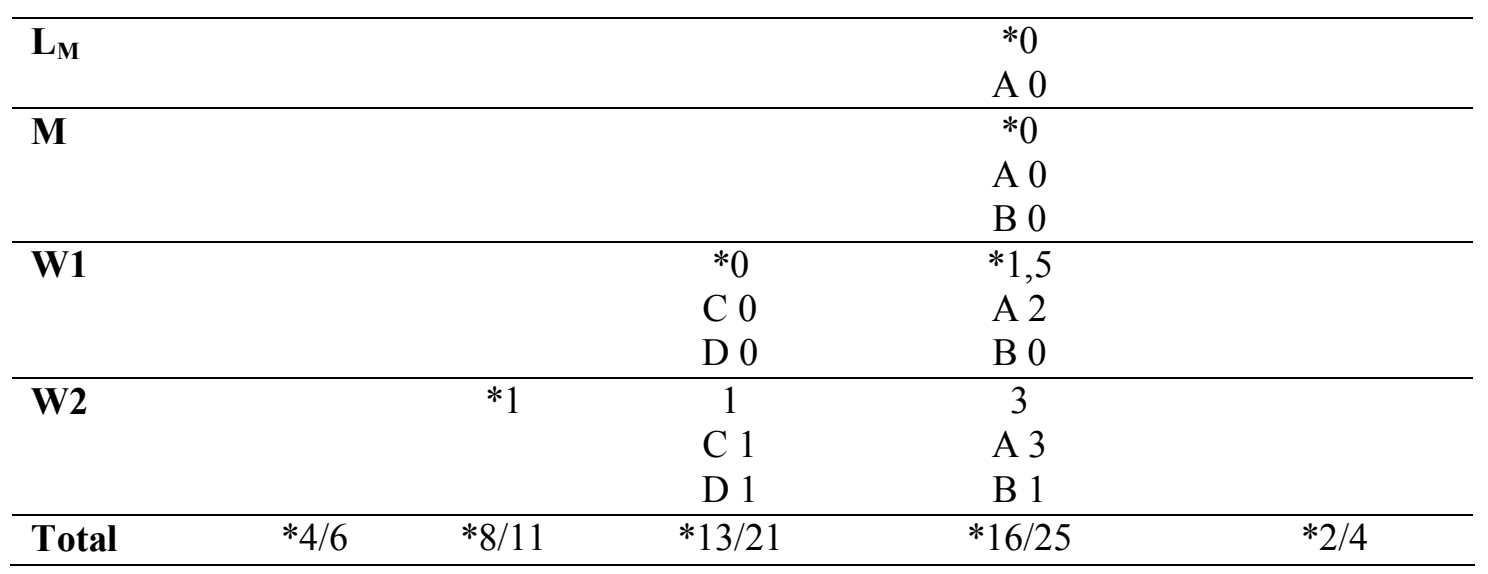

\section{Ecological status assessment}

The assessement of ecological status was based on main and additional phytoplankton metrics (Table 6). Individual class boundaries were in concordance with scales for oligo and mesotrophic lake types (Table 1 and Table 2). Both water bodies in Lake Subgroup I.1 (Alpine lakes) were in reference conditions. Three oligotrophic mountain reservoirs (Lake Group I) were also in high status, except for AGI and transparency values at Toshkov chark Reservoir.

High to moderate status dominated within Lake Group II. Srechenska bara drinking water reservoir was evaluated in high status based on all metrics. Q index, AGI and transparency resulted in one class lower status in comparison with biomass at Kardzhali Reservoir. Reservoirs Studen kladenets, Ivaylovgrad and Devets, under the urban impact were in good status after main metrics (Table 6), except for $\mathrm{Q}$ index at Ivaylovgrad Reservoir coincided with first degree bloom of Oocystis solitaria $\left(1.4 \mathrm{mg} \mathrm{l}^{-}\right.$ 1). At Krichim Reservoir separate metrics differed, but biomass and Q index revealed good status. AGI, transparensy and chlorophyll $a$ were in lower ranges due to bloom of colonial diatom Fragilaria crotonensis (4.1 $\mathrm{mg}^{-1}-$ second degree). Reservoirs Borovista and Vacha, under eutrophication, had moderate status, since metrics reflecting species composition (Q, AGI and \% Cyanobacteria) are more sensitive than biomass. Diatom Aulacoseira granulata (2.96 $\mathrm{mg} \mathrm{l}^{-1}$ - II degree) bloomed at Borovista Reservoir. Metric \% Cyanobacteria (33.6) was increased due to eutrophic Aphanizomenon flos-aquae at Vacha Reservoir. Both indices resulted in lower status then biomass in half of the water bodies within the group.

Lake Group III included Eleshnitsa Reservoir in reference conditions and Poroj Reservoir in good status. Ovcharitsa Reservoir was assessed in moderate status based on bloom of colonial diatom Aulacoseira granulata (2.39 $\mathrm{mg}^{-1}-$ II degree) and green flagellat Carteria multifilis (0.96 mg $1^{-1}-$ I degree). Durankulak swamp was evaluated in poor status, regardless most main metrics average levels. High \% Cyanobacteria $(25.56 \%)$ and second degree bloom of Microcystis aeruginosa $\left(2.65 \mathrm{mg} \mathrm{l}^{-1}\right)$ were registered. Acheloy Reservoir was in bad status in the view of Q index, AGI and exclusively high percent eutrophic Cyanobacteria (92\%) from FGs S1 (Limnothrix redekei, Pseudanabaena limnetica) and $\mathbf{H 1}$ (Aphanizomenon flos-aquae) - Table 4.

Hyperhaline Atanasovsko Lake (Lake Group IV) had contradictory evaluation after applied metrics. Biomass, Q index and second degree bloom of green flagellats Pyramimonas tetrarhynchus $\left(4.43 \mathrm{mg}^{-1}\right)$ and Provasoliella ovata $\left(6.53 \mathrm{mg} \mathrm{l}^{-1}\right)$ resulted 
in moderate status. AGI was neglected as unrepresentative metric since does not enlisted green flagellats, as well as metric \% Cyanobacteria indicative of freshwater ecosystems.

Table 6. Ecological status of the lakes according to phytoplankton. Legend: Ltc-Lake Type Code; Bm-Biomass; Q - Assemblage Index; AGI-Algae Group Index; EQR - ecological quality ratio; $S D$ - Transparensy; Chl a - Chlorophyll a; \% Cyano - \% Cyanobacteria; $B i-$ "Bloom" (intensity); Chl-Chlorophyceae; Ba-Bacillariophyceae; $C y-$

Cyanoprokaryota; Bts - "Bloom” (toxic species); ES - Ecological Status; H-high; Ggood; $M$ - moderate; $P$ - poor; $B$ - bad.

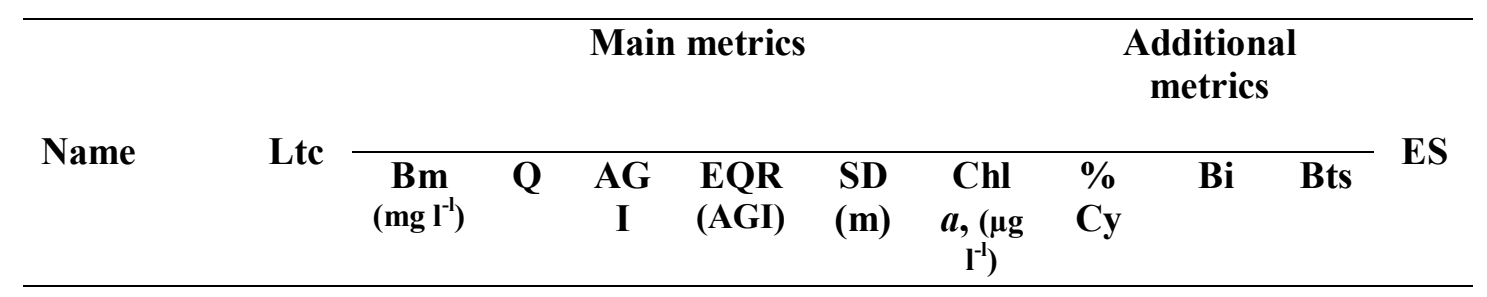

Lake Subgroup I.1 - Alpine lakes

\begin{tabular}{llllllclllll}
\hline Chernoto & L1 & 0.57 & 4.9 & 0.55 & 0.999 & $\begin{array}{c}> \\
15.5\end{array}$ & $<0.2$ & 0.00 & no & no & H \\
\hline Bezbog & L1 & 0.95 & 4.5 & 0.48 & 0.998 & $\begin{array}{c}> \\
7.0\end{array}$ & $<0.2$ & 0.74 & no & no & H \\
\hline
\end{tabular}

Lake Group I - Mountain „lakes”

\begin{tabular}{lccccccccccc}
\hline Belmeken & L13 & 0.79 & 4.9 & 0.51 & 0.999 & 4.0 & 2.60 & 0.00 & no & no & H \\
\hline Beli Iskar & L1 & 0.76 & 4.7 & 0.57 & 0.999 & 6.5 & 1.15 & 0.00 & no & no & H \\
\hline $\begin{array}{l}\text { Toshkov } \\
\text { chark }\end{array}$ & L3 & 0.18 & 4.1 & 1.04 & 0.998 & 3.5 & 2.20 & 0.00 & no & no & H \\
\hline
\end{tabular}

Lake Group II - Deep semi mountain and lowland "lakes"

\begin{tabular}{lcccccccccccc}
\hline $\begin{array}{l}\text { Srechenska } \\
\text { bara }\end{array}$ & L2 & 1.01 & 5.0 & 0.40 & 0.999 & 4.5 & 1.34 & 0.00 & no & no & H \\
\hline Kardzhali & L11 & 0.42 & 3.9 & 1.27 & 0.997 & 3.6 & 1.82 & 0.00 & no & no & H \\
\hline $\begin{array}{l}\text { Studen } \\
\text { kladenets }\end{array}$ & L11 & 1.16 & 3.6 & 1.04 & 0.998 & 2.4 & 2.44 & 0.00 & no & no & G \\
\hline Ivaylovgrad & L11 & 2.02 & 2.8 & 0.92 & 0.998 & 2.6 & 4.02 & 2.18 & I Chl & no & G \\
\hline Devets & L16 & 2.44 & 3.6 & 1.73 & 0.996 & 2.0 & 4.07 & 0.00 & no & no & G \\
\hline Krichim & L11 & 4.77 & 3.2 & 8.37 & 0.979 & 1.1 & 14.50 & 0.00 & II Ba & no & G \\
\hline Borovitsa & L13 & 4.37 & 2.9 & 6.04 & 0.985 & 1.5 & 6.93 & 0.14 & II Ba & no & M \\
\hline Vacha & L11 & 2.29 & 1.6 & 6.24 & 0.985 & 1.3 & 3.50 & 33.6 & no & yes & M \\
\hline
\end{tabular}

Lake Group III - Small and middle sized lowland "lakes"

\begin{tabular}{ll|llllllllllll} 
Eleshnitsa & L12 & 0.48 & 4.1 & 1.17 & 0.997 & 1.2 & 0.98 & 0.00 & no & no & H \\
\hline
\end{tabular}




\begin{tabular}{lcccccccccccc}
\hline Poroj & L16 & 1.77 & 3.6 & 1.03 & 0.998 & 1.10 & 3.56 & 5.08 & no & no & G \\
\hline Ovcharitsa & L12 & 6.37 & 2.6 & 1.2 & 0.997 & 2.3 & 7.32 & 2.97 & II Ba & yes & M \\
& & & & & & & & & & & \\
\hline Durankulak & L7 & 7.63 & 2.3 & 6.72 & 0.983 & 0.43 & 15.63 & $\begin{array}{c}25.5 \\
6\end{array}$ & II Cy & yes & P \\
\hline Acheloy & L16 & 14.28 & 0.4 & 44.1 & 0.890 & 0.65 & 27.5 & 92.1 & III & yes & B \\
& & & & & & & & & Cy & & \\
\hline
\end{tabular}

Lake Group IV - Black Sea hyperhaline coastal lakes

\begin{tabular}{lllllllllllllll}
\hline Atanasovsko & L10 & 12.47 & 2.9 & 0.4 & 0.999 & 0.25 & 31.2 & 0.00 & $\begin{array}{c}\text { II } \\
\text { Chl }\end{array}$ & no & ?M \\
\hline
\end{tabular}

Both main and additional phytoplankton metrics showed correlation, as Q index and AGI were highly correlated (Table 7). Assemblage index had also strong relationship $(\mathrm{p}<0.001)$ with Total biomass, \% Cyanobacteria, Chlorophyll $a$, as well as with the assessed overall ecological status (ES).

Table 7. Correlation matrix for all 8 metrics at four groups lakes. Level of significance: *** $-p<0.001, * *{ }_{-} p<0.01, *{ }_{-} p<0.05$.

\begin{tabular}{|c|c|c|c|c|c|c|c|c|}
\hline & AGI & $\begin{array}{c}\text { EQR } \\
\text { (AGI) }\end{array}$ & $\mathbf{Q}$ & Q Status & Biom ass & Chl $a$ & $\%$ Cy & ES \\
\hline AGI & & $-1.00^{* * * *}$ & $-0.73^{* *}$ & $0.71 * *$ & $0.87^{* * *}$ & $0.87 * * *$ & $0.94 * * *$ & $0.76 * * *$ \\
\hline $\begin{array}{l}\text { EQR } \\
\text { (AGI) }\end{array}$ & & & $0.73 * *$ & $-0.71 * *$ & $-0.88 * * *$ & $-0.88^{* * *}$ & $-0.94 * * *$ & $-0.77 * * *$ \\
\hline Q & & & & $-0.97 * * *$ & $-0.82 * * *$ & $-0.75^{* * *}$ & $-0.79 * * *$ & $-0.88 * * *$ \\
\hline $\mathbf{Q}$ & & & & & $0.80^{* * *}$ & $0.70^{* *}$ & $0.78^{* * *}$ & $0.85^{* * *}$ \\
\hline Status & & & & & & & & \\
\hline B m & & & & & & $0.96 * * *$ & $0.83^{* * *}$ & $0.92 * * *$ \\
\hline Chl $a$ & & & & & & & $0.80 * * *$ & $0.84 * * *$ \\
\hline$\% \mathrm{Cy}$ & & & & & & & & $0.83^{* * *}$ \\
\hline
\end{tabular}

\section{Discussion}

\section{Assemblage index ( $Q$ index)}

\section{Functional groups (codons)}

Species from functional assemblages $\mathbf{L}_{\mathbf{0}}, \mathbf{Y}$ and $\mathbf{M P}$ were first and second biomass dominants at ultra oligotrophic alpine lakes (Table 4). Codons $\mathbf{L}_{\mathbf{0}}$ (mostly dinoflagellates) and Y (large size cryptomonads) occur in broad variety of habitats and are able to survive in all lentic ecosystems conditions (Padisák et al. 2009, Kruk et al. 2010). Our study confirmed these groups were specific not only for alpine lakes. As dominant species from Codon $\mathbf{L}_{\mathbf{0}}$ were registered also at mountain Belmeken and Beli Iskar Reservoirs, deep semi mountain and lowland Srechenska bara, Kardzhali and Devets Reservoirs and lowland Eleshnitsa Reservoir (Table 4). Since all the above water bodies were in high or good status, we assumed that Codon $\mathbf{L}_{\mathbf{0}}$ included reference species for national types L1, L2, L11, L12, L13 and L16. Species from Codon Y were also registered at all lake groups but in biomass under 5\% (Table 4 and Table 5). Codon 
MP was suggested to unify all the meroplanktonic autotrophic organisms that can be accidentally found in phytoplankton samples, independently of lake type (Padisák et al. 2009). During our study we found that MP species were dominant also in deep oligotrophic semi mountain Studen kladenets and Devets Reservoirs (Table 4). Assessed high and good status demonstrated that codon MP can also be applied as indicative for undisturbed conditions.

At three oligotrophic mountain lakes in reference conditions, dominant species were from assemblage $\mathbf{L}_{\mathbf{0}}, \mathbf{N}, \mathbf{E}$ and $\mathbf{C}$ (Table 4 and Table 6). Codon $\mathbf{N}$ (planctonic desmids and diatom Tabellaria) is related to summer season in unimpacted lakes in continental zone (Reynolds et al. 2002). Our results showed codon $\mathbf{N}$ was dominant in epilimnion of deep mountain Belmeken Reservoir. Moreover $\mathbf{N}$ was established at other lake types such as deep semi mountain Devets and lowland Eleshnitsa Reservoir, all reservoirs in high or good status (Table 4 and Table 6). Codon E (Chrysophyceans - silica scaled flagellates) developes usually in small, shallow, base poor lakes or heterotrophic ponds (Reynolds et al. 2002). Species are tolerant to low nutrients and sensitive to $\mathrm{CO}_{2}$ deficiency. Codon $\mathbf{E}$ was registered in high relative abundance at mountain Toshkov chark Reservoir (Table 4), which is a natural dystrophic, peaty lake.

Eight water bodies were selected among deep semi mountain and lowland lakes. Their ecological status varied from high to moderate, and first and second dominants were taxa from codons $\mathbf{L}_{\mathbf{0}}, \mathbf{X 3}, \mathbf{B}, \mathbf{F}, \mathbf{J}, \mathbf{P}$, and $\mathbf{H 1}$ (Table 4). Based on frequency distribution of the FG, two reservoirs groups were outlined. First group included Studen kladenets, Kardzhali and Ivaylovgrad Reservoirs with first dominant species from codon F (mucilaginous colonial green algae). The assemblage is character for oligotrophic epilimnion, tolerant to low nutrients, but susceptible to shaded habitats (Reynolds et al. 2002). Our study confirmed codon $\mathbf{F}$ is characteristic to wide range of unimpacted lakes, but is with high relative abundance mainly in deep oligotrophic lake indicating good status (Table 4 and Table 6). Second reservoir group (Borovitsa, Krichim and Devets) was situated at higher altitude $(419-529 \mathrm{~m})$, and had lower transparency (SD: 1.1-1.5 m). Obviously they were under the impact of stronger eutrophication and dominant was codon $\mathbf{P}$ (colonial diatoms and desmids), typical to eutrophic epilimnnia (Reynolds et al., 2002). Colonial diatom Fragilaria crotonensis was monodominant with $85.5 \%$ from the total biomass at Krichim Reservoir. In Borovitsa Reservoir Aulacoseira granulata accounted for $67.8 \%$ of the biomass, while unicells desmids Staurastrum teliferum and Staurastrum gracile for half of the biomass in Devets Reservoir. Assemblage $\mathbf{P}$ high relative abundance is indicative for ecological status alteration towards moderate conditions at deep semi mountain reservoirs.

Small and middle sized lowland lakes covered a shallow, polimictic swamp and 4 middle deep, stratified in summer reservoirs. Amnog twenty-five FG presented, specific for the particular lake group were codons $\mathbf{L}_{\mathbf{M}}, \mathbf{X}_{\mathbf{P h}}, \mathbf{K}, \mathbf{T}, \mathbf{M}, \mathbf{S 2}$. Various athropogenic pressures influenced dominant species and assemblages (Table 4 and Table 6). Reference Eleshniza Reservoir had as dominant codon $\mathbf{X 3}$, which grows in oligotrophic conditions, presented by Stichococcus minutissimum. Chlamydomonas sp. was first dominant at Poroj Reservoir, from mesotrophic assemblage X2, typical to shallow, clear mixed layers in meso-eutrophic lakes (Reynolds et al., 2002). Ceratium furcoides from codon $\mathbf{L}_{\mathbf{M}}$, together with Microcystis aeruginosa dominated at Ovcharitsa Reservoir. At the assemblage Ceratium was associated with Microcystis, and reflected higher trophic status, based on eutrophic to hypertrophic, small to middle sized habitat pattern of $\mathbf{L}_{\mathbf{M}}$. Thus three codons, single or together with Ceratium and Microcystis development, 
connection with eutrophication trend $\mathbf{L}_{\mathbf{0}} \rightarrow \mathbf{L}_{\mathbf{M}} \rightarrow \mathbf{M}$ reported previously by Padisák et al. (2009) was confirmed. At Durankulak swamp codon $\mathbf{J}$ was established and accounted for a half of the biomass (Table 4). In conformity with exsiting habitat description from Reynolds et al. (2002), the group inhabited shallow, mixed, with high nutrient content waters. Group M (Microcystis aeruginosa) was registered at these eutrophic conditions. At Acheloy Reservoir in eutrophic conditions and low transparency $(0.6 \mathrm{~m})$, dominants were shade-adapted cyanoprokaryotes from codon $\mathbf{S 1}$ (Limnothrix redekei and Pseudanabaena limnetica), which formed more than $80 \%$ of the biomass (Table 4). High S/V proportion determined their tolerance to limited light availability (Naselli-Flores and Barone, 2007). Eutrophic assemblage H1 (dinitrogenfixing Nostocaleans) was subdominant and incorporated Aphanizomenon flos-aquae.

Studied modified section of Atanasovsko Lake was shallow, polimictic, with high salinity $(63.4 \%)$ and conductivity $\left(105,003 \mu \mathrm{S} \mathrm{cm} \mathrm{cm}^{-1}\right)$. Almost $90 \%$ of biomass was formed by X2 (Table 4). Both Provasoliella ovata and Pyramimonas tetrarhynchus bloomed intensively during the study (Table 6). Provasoliella ovata refers to birdmanured pools and other organically enriched waters (John et al., 2003). The registered boom probably can be related to natural organic enrichment from birds and lack of specialized zooplankton. Padisák et al. (2003) reported dominance of X2 (as well as Y) in zooplankton lacing habitats.

The analysis of FG frequency distribution showed high relative abundance of assemblages $\mathbf{L}_{\mathbf{0}}, \mathbf{Y}, \mathbf{M P}, \mathbf{N}, \mathbf{E}, \mathbf{X} \mathbf{3}, \mathbf{X}_{\mathbf{P h}}$ (depedant on lake type) indicated high or good status. At oligotrophic stratified reservoirs and naturally mezoeutrophic lowland lakes undesirable assemblages were $\mathbf{L}_{\mathbf{M}}, \mathbf{J}, \mathbf{M}, \mathbf{S 1}$ and $\mathbf{H 1}$.

\section{Factor number $(F)$}

Factor number (F) pre-determination according to the existing typology is the most crucial step, which defines the index (Q) impartial assessment (Padisák et al., 2006; Crossetti and Bicudo, 2008; Becker et al., 2009; 2010). Following the existing knowledge of Q implementation, we defined F for each FG. Thus higher F values were allocated to FGs typical of pristine conditions, whereas lower values were set for undesirable ones.

Since $\mathrm{F}$ has to be specified for each lake type, the lack of paleolimnological data for most of the national types caused perplexity. Major emphasis was given on to floristic and taxonomic surveys in Bulgaria (Vodenicharov and Vodenicharov, 2000).

Determination of F per FGs in Alpine lakes followed background for seven glacial lakes (Beshkova, 2000). Dominant species reported during 1995 - 1996, belonged to the same groups established in the current study: $\mathbf{L}_{\mathbf{0}}, \mathbf{E}, \mathbf{Y}$ and additionaly $\mathbf{N}$. This finding verified reference character of the above groups for summer period in Bulgarian glacial lakes. Vodenicharov and Vodenicharov (2000) reported dominance of Bacillariophyceae and Desmidiales in such lentic ecosystems. Species from Chlorococcales appeared also, indicating eutrophication mainly due to touristic activities. Based on that FG $\mathbf{F}$ received the lowest value 2 of Factor number (Table 5).

Factor number at Lake Group II (Deep semi mountain and lowland lakes) was based on Becker et al. (2009; 2010) researched Faxinal Reservoir and Sau Reservoir. Similar to them is national lake type L11 (Large deep reservoirs), included in Lake Group II. Regardless of similarities we took into account that $\mathrm{F}$ values have to be set according to the typology (Padisák et al., 2006) and adapted F as followed: P - 3; B - 5; F $-3 ; \mathbf{L}_{\mathbf{0}}-5$ 
and X1 - 2,5 (Table 5). Adaptation was based on Q calculation for reservoirs at which all phytoplankton metrics resulted in equal ecological status assessment. For example at Devets Reserervoir all basic metrics determined good ecological status (Table 6). Simulatneously if F values cited by Becker et al. $(2009 ; 2010)$ were applied, Q index assessed unadequate lower status.

Lake Group III (Small and middle sized lowland lakes) was compared to F rates for Hungarian type 7 after Padisák et al. (2006) and shallow Garças Reservoir after Crossetti and Bicudo (2008), which appeared most similar to our national types. Calibration of $\mathrm{F}$ was based on Poroj Reservoir, which was in good ecological status according Biomass, AGI and \% Cyanobacteria (Table 6).

Factor number calibration was complicated at Lake Group IV (Black Sea hyperhaline coastal lakes). Atanasovsko Lake has been artificialy modified and there are no paleolimnological records before modifications. Thus $\mathrm{F}$ values were based totally on species autecology, i.e. flagellates green algae Provasoliella ovata distribution in small nutrient rich lakes (John et al., 2003). Therefore F $=3$ was determined for group X2 which was formed up to $90 \%$. Since no autecological data were available for the rest of the species, the particular groups received the same F values as in Lake Group III, but these rates need further verification.

\section{Algae Group Index (AGI, Catálan Index)}

Several groups such as Zygnemaphyceae, Euglenophyceae and single-celled green flagellates (Order Polyblepharidales, Tetraselmidales, Chlamydomonadales) are not included in AGI calculation. High coefficient of Cyanobacteria illustrates that AGI assess mainly eutrophication.

\section{Comparative assessment of ecological status based on two indices}

Both indices gave equal assessmet in four lakes within the first Lake Group - Alpine and Mountain lakes (Table 6). All metrics illustrated ultraoligo- and oligotrophic regerence conditions except for Toshkov chark Reservoir evaluated in good status according to AGI. Q index and AGI evaluated 5 lakes in identical status within Lake Group II - Deep semi mountain and lowland lakes, similar to common European L-M7 type. Both indices assessed lower status in four cases in contrast to Total biomass, which confirmed that Q index evaluates an ecological status one category lower than the classical biomass-based qualification (Padisák et al., 2006). Three cases of equal status assessed by two indices were found at third Lake Group - Small and middle sized lowland lakes.

In general, within Lake Groups I, II and III, Q index and AGI had good correlation in assessment. In non-conformity with biomass, two indices qualified lower category lake status, since they are based not only on biomass, but on phytoplankton taxa, thus they are more sensitive to changes in species composition.

AGI cannot be applied properly in Lake Group IV - Black Sea hyperhaline coastal lakes, where single-celled green flagellates consisted up to $90 \%$ of the total biomass and are not included in the AGI calculation. According Becker et al. (2009), because of high sensitivity of Q to species appearance, it can give realistic evaluation of ecological status, especially in ecosystems with specific conditions, such as haline lakes or naturaly eutrophic lakes with increased phosphorus levels. Moreover, AGI is not recommended in lentic ecosystems with excessed Euglenophyceae growth, e.g. riverine 
marshes (Bulgarian L5 type; unpublished data).

\section{Conclusion}

Assemblage index and Algae Group Index resulted in similar assessment and were applicable to all three Alpine and mountain, Deep semi-mountain and Small and middle sized lowland lake groups. Assemblage index is recommended especially for lentic ecosystems in extreme cases, e. g. hyperhaline lakes, for its flexibility and more adequate assessment. Important taxonomic groups, which can be dominant to biomass in particular lake types and which are not included in AGI, determined index restricted applicability. There is no constraint on the Q index based assessment except that the F number has to be correctly determined for each lake type. When paleolimnological data are missing, empirical approach to calibrate $\mathrm{F}$ based on lakes under reference conditions can be recommended.

Acknowledgments. This work was supported by EU funded project under Operational Programme "Environment" 2007-2013, Priority Axis I: "Development of a classification system for assessing the ecological status and ecological potential of the determined types of surface waters (rivers and lakes) on the territory of the Republic of Bulgaria (on the basis of a system "B" typology)".

\section{REFERENCES}

[1] Agència Catalana de l'Aigua. (2003): Desenvolupament d'un índex integral de qualitat ecològica i regionalització ambiental dels sistemes loacustres de Catalunya. - Generalitat de Catalunya, Department de Medi Ambient i Habitatge.

[2] Becker, V., Huszar, V.L.M., Crossetti, L.O. (2009): Responses of phytoplankton functional groups to the mixing regime in a deep subtropical reservoir. - Hydrobiologia 628: 137-151.

[3] Becker, V., Caputo, L., Ordóñez, J., Marcé, R., Armengol, J., Crossetti, L.O., Huszar, V. (2010): Driving factors of the phytoplankton functional groups in a deep Mediterranean reservoir. - Water Research 44: 3345-3354.

[4] Beshkova, M. (2000): The phytoplankton of the glacial high mountain lakes Sedemte Rilski ezera (the Rila Mountains, Bulgaria). - In: Golemansky, V., Naidenow, W. (eds) Biodiversity of Shabla lake system, Instit. Zoolog. Min. Environ. Wat., Sofia.

[5] Cardoso, A.C., Duchemin, J., Margarou, P., Premazzi, G. (2001): Criteria for the identification of freshwaters subject to eutrophication. Their use for implementation of the "Nitrates" and Urban Waste Water Directives. Environment Institute, Water research and Monitoring Unit, JRC Ispra and Directorate General for Environment, European Commission, EUR 1910 EN.

[6] Cheshmedjiev, S., Belkinova, D., Mladenov, R., Dimitrova-Dyulgerova, I., Gecheva, G. (2010a): Phytoplankton based assessment of the ecological status and ecological potential of lake types in Bulgaria. - Biotechnology and Biotechnological Equipment 24: 14-25.

[7] Cheshmedjiev, S., Karagiozova, T., Michailov, M., Valev, V. (2010b): Revision of river and lake typology in Bulgaria within ecoregion 12 (Pontic Province) and ecoregion 7 (Eastern Balkans) according to the Water Framework Directive. - Ecologia Balkanica 2: 75-96.

[8] Cole, G.A. (1994): Textbook of Limnology. - Waveland Press, Illinois. 
[9] Crossetti, L.O., Bicudo, C.E.M. (2008): Phytoplankton as a monitoring tool in a tropical urban shallow reservoir (Garças Pond): the assemblage index application. Hydrobiologia 610: 161-173.

[10] Dokulil, M., Teubner, K. (2006): Bewertung der Phytoplanktonstruktur stehender Gewässer gemäß der EU-Wasserrahmenrichtlinie: Der modifizierte Brettum Index. - In: DGL-Tagungsbericht 2005, Karlsruhe.

[11] EC Parliament and Coincil. (2000): Directive of the European Parliament and of the Council 2000/60/EC Establishing a Framework for Community Action in the Field of Water Policy. - Official Journal of European Union L 327: 1-72.

[12] Hillebrand, H., Dürseken, D., Kirschtel, D., Pollingher, U., Zohary, T. (1999): Biovolume calculation for pelagic and benthic microalgae. - Journal of Phycology 35: 403-424.

[13] Jeffrey, S.W., Humphrey, G.F. (1975): New spectrophotometric equations for chlorophylls a, b, c1 and c2 in higher plants, algae and natural phytoplankton. - Journal of Plant Physiology 167: 191-194.

[14] John, D.M., Whitton, B.A., Brook, A.J. (2003): The Freshwater Algal Flora of the British Isles. An Identification Guide to Freshwater and Terrestrial Algae. - Cambridge University Press, Cambridge.

[15] Lund, J.W.G., Kipling, C., Lecren, E.D. (1958): The inverted microscope method of estimating algal number and the statistical basis of estimating by counting. Hydrobiologia 11: 143-170.

[16] Marchetto, A., Padedda, B.M., Mariani, M.A., Lugliè, A., Sechi, N. (2009): A numerical index for evaluating phytoplankton response to changes in nutrient levels in deep mediterranean reservoirs. - Journal of Limnology 68: 106-121.

[17] Kruk, C., Huszar, V.L.M., Peeters, E.T.H.M., Bonilla, S., Costa, L., Lürling, M., Reynolds, C., Scheffer, M. (2010): A morphological classification capturing functional variation in phytoplankton. - Freshwater Biology 55: 614-627.

[18] Naselli-Flores, L., Barone, R. (2007): Pluriannual morphological variability of phytoplankton in a highly productive Mediterranean reservoir (Lake Arancio, Southwestern Sicily). - Hydrobiologia 578: 87-95.

[19] Padisák, J., Borics, G., Fehér, G., Grigorszky, I., Oldal, I., Schmidt, A., Zámbóné-Doma, Z. (2003): Dominant species, functional assemblages and frequency of equilibrium phases in late summer phytoplankton assemblages in Hungarian small shallow lakes. Hydrobiologia 502: 157-168.

[20] Padisák, J., Borics, G., Grigorszky, I., Soróczki-Pintér, É. (2006): Use of phytoplankton assemblages for monitoring ecological status of lakes within the Water Framework Directive: the assemblage index. - Hydrobiologia 553: 1-14.

[21] Padisák, J., Crossetti, L.O., Naselli-Flores, L. (2009): Use and misuse in the application of the phytoplankton functional classification: a critical review with updates. Hydrobiologia 621: 1-19.

[22] Philippe, M., Vey, V., Barbe, J. (2003): Actualisation de la méthode de diagnose rapide des plans d'eau. Analyse critique des indexes de qualité des lacs et propositions d'indexes de fonctionnement de l'ecosystème lacustre. - Cemagref, Lyon.

[23] Phillips, G., Morabito, G., Carvalho, L., Solhein, A.L., Skjelbred, B., Moe, J., Andersen, T., Mischke, U., de Hoyos, C., Borics, G. (2011): Report on lake phytoplankton composition metrics, including a common metric approach for use in intercalibration by all GIGs. - WISER deliverable D3.1.1, http://www.wiser.eu/download/D3.1-1_draft.pdf.

[24] Reynolds, C.S., Huszar, V.L.M., Kruk, C., Naselli-Flores, L., Melo, S. (2002): Towards a functional classification of the freshwater phytoplankton. - Journal of Plankton Research 24: $417-428$. 
[25] Salmaso, N., Morabito, G., Buzzi, F., Garibaldi, L., Simona, M., Mosello, R. (2006): Phytoplankton as an indicator of the water quality of the deep lakes south of the Alps. Hydrobiologia 563: 167-187.

[26] Salmaso, N., Padisák, J. (2007): Morpho-functional groups and phytoplankton development in two deep lakes (Lake Garda, Italy and Lake Stechlin, Germany). Hydrobiologia 578: 97-112.

[27] Sirenko, L.A., Gavrilenko, M.Y. (1978): Water Blooms and Eutrophication. - Naukova dumka, Kiev (in Russian).

[28] Strickland, J.D.H., Parsons, T.R. (1972): A practical handbook seawater analysis. Bulletin of the Fisheries Research Board of Canada, Ottawa.

[29] Ter Braak, C.J.F., Šmilauer, P. (2002): CANOCO reference manual and CanoDraw for Windows user's guide: software for canonical community ordination (version 4.5). Microcomputer Power, Ithaca, New York.

[30] Utermöhl, H. (1958): Zur Vervollkommnung der quantitativen Phytoplankton-Methodik. Mitteilungen der internationale Vereinigung der theoretische und angewandte. Limnologie 5: 567-596.

[31] Vodenicharov, D., Vodenicharov, M. (2000): Biodiversity of fresh water algae in the Rila National Park. - In: Sakalian, M. (ed.) Biological diversity of the Rila National Park. Pensoft, Sofia.

[32] Wetzel, R.G., Likens, G.E. (2000): Limnological Analyses - Springer-Verlag, New York Inc., New York. 